Zbigniew Grande*, Institute of Mathematics, Pedagogical University, Plac Weyssenhoffa 11, 85-072 Bydgoszcz, Poland. e-mail:

grande@wsp. bydgoszcz.pl

\title{
ON A. C. LIMITS OF DECREASING SEQUENCES OF CONTINUOUS OR RIGHT CONTINUOUS FUNCTIONS
}

\begin{abstract}
The a.c. limits (i.e. the discrete limits introduced by Császár and Laczkovich) of decreasing sequences of continuous (resp. right continuous) functions are investigated.
\end{abstract}

Let $\mathbb{R}$ be the set of all reals. $(X, \tau)$ or $X$ in this paper always denotes a perfectly normal Hausdorff topological space. A function $f: X \rightarrow \mathbb{R}$ is a $B_{1}^{*}$ function (belongs to the class $B_{1}^{*}$ ) if there is a sequence of continuous functions $f_{n}: X \rightarrow \mathbb{R}$ with $f=$ a.c. $\lim _{n \rightarrow \infty} f_{n}$, i.e. for each point $x \in X$ there is a positive integer $k$ such that $f_{n}(x)=f(x)$ for every $n>k$ (compare [2, 3]).

From the results obtained in [2] it follows that the function $f: X \rightarrow \mathbb{R}$ belongs to $\mathcal{B}_{1}^{*}$ if and only if there are closed sets $A_{n}, n=1,2, \ldots$, such that the restricted functions $f \uparrow A_{n}$ are continuous and $X=\bigcup_{n=1}^{\infty} A_{n}$.

\section{The Discrete Limits of Decreasing Sequences of Con- tinuous Functions.}

In the first part of this article we will investigate $B_{1}^{*}$ functions which are upper semicontinuous. Recall that the function $f: X \rightarrow \mathbb{R}$ is upper semicontinuous if for every real $a$ the set $\{x \in X ; f(x)<a\}$ belongs to $\tau$. Evidently the pointwise limit of each decreasing sequence of upper semicontinuous functions $f_{n}: X \rightarrow \mathbb{R}, n=1,2, \ldots$, is upper semicontinuous.

The following theorem can be found on page 51 of [4].

Key Words: upper semicontinuity, decreasing sequences of functions, $B_{1}^{*}$ class, right continuity.

Mathematical Reviews subject classification: 26A15, 26A21, 26A99

Received by the editors August 3, 1998

*Supported by Bydgoszcz Pedagogical University grant 1998 
Remark 1. If the function $f: X \rightarrow \mathbb{R}$ is upper semicontinuous, then there is a decreasing sequence of continuous functions $f_{n}: X \rightarrow \mathbb{R}, n=1,2, \ldots$, such that $f=\lim _{n \rightarrow \infty} f_{n}$.

We will prove the following theorem.

Theorem 1. Let $(X, \tau)$ be a perfectly normal $\sigma$-compact Hausdorff topological space. Then an upper semicontinuous function $f: X \rightarrow \mathbb{R}$ belongs to class $B_{1}^{*}$ if and only if there is a decreasing sequence of continuous functions $f_{n}: X \rightarrow \mathbb{R}$ such that $f=$ a. c. $\lim _{n \rightarrow \infty} f_{n}$.

We start from the following lemma.

Lemma 1. Let $f: X \rightarrow \mathbb{R}$ be a function. If there are sets $A_{n}$ and continuous functions $f_{n}: X \rightarrow \mathbb{R}$ such that $A_{1} \subset A_{2} \subset \cdots, X=\bigcup_{n} A_{n}, f_{n} \geq f$ and $f_{n}\left\lceil A_{n}=f\left\lceil A_{n}\right.\right.$ for $n=1,2, \ldots$, then there is a decreasing sequence of continuous functions $g_{n}: X \rightarrow \mathbb{R}$ with $f=$ a. c. $\lim _{n \rightarrow \infty} g_{n}$.

Proof. Of course, the functions $g_{n}=\min _{k \leq n} f_{n}$ satisfy all required conditions.

Proof of Theorem 1. If $f$ is the discrete limit of a decreasing sequence of continuous functions $f_{n}: X \rightarrow \mathbb{R}$, then evidently $f \in \mathcal{B}_{1}^{*}$. So, we assume that $f \in \mathcal{B}_{1}^{*}$. Since $f$ is upper semicontinuous and $X$ is perfectly normal, by Remark 1 there is a decreasing sequence of continuous functions $f_{n}: X \rightarrow \mathbb{R}$ which converges to $f$ at each point $x \in X$.

On the other hand $f$ is the discrete limit of continuous functions; so there are closed sets $A_{n}, n=1,2, \ldots$, such that every restricted function $f\left\lceil A_{n}\right.$ is continuous and $X=\bigcup_{n=1}^{\infty} A_{n}$. We can assume that $A_{n}$ is compact for each $n=1,2, \ldots$. Fix a positive integer $k$. On $A_{k}$ the sequence $\left(f_{n}\right)$ tends uniformly to $f$ due to Dini's lemma. So we can also assume that

$$
\max \left\{\left(f_{n}(x)-f_{n+1}(x)\right) ; x \in A_{k}\right\} \leq 2^{-n} .
$$

By Tietze's theorem for $n=1,2, \ldots$ there is a continuous extension $g_{n}: X \rightarrow$ $\left[0,2^{-n}\right]$ of the restricted function $\left(f_{n}-f_{n+1}\right) \uparrow A_{k}$. Let

$$
h_{n}=\min \left(g_{n}, f_{n}-f_{n+1}\right) \text { for } n=1,2, \ldots,
$$

and let $l_{k}=f_{1}-\sum_{n=1}^{\infty} h_{n}$. Since the series $\sum_{n=1}^{\infty} h_{n}$ converges uniformly, the function $l_{k}$ is continuous. Moreover, for $k=1,2, \ldots$ we have $l_{k} \geq f$ and $f \uparrow A_{k}=l_{k} \uparrow A_{k}$. So, by Lemma 1 we obtain our theorem.

Theorem 1 in the presented form and its proof was proposed by the referee. My formulation concerned the function $f:[a, b] \rightarrow \mathbb{R}$ and the Euclidean topology and its proof was more complicated. 


\section{Decreasing Sequences of Right Continuous Functions}

In this part we assume that $X=[a, b)$ and $\tau$ is the topology of right continuity. This topology $\tau$ is perfectly normal and Hausdorff but is not $\sigma$-compact. So, the limit $f$ of a decreasing sequence of right upper semicontinuous functions $f_{n}, n=1,2, \ldots$, is a right upper semicontinuous function and Remark 1 is valid for $(X, \tau)$. Thus we have the following assertion.

Remark 2. For every right upper semicontinuous function $f$ there is a decreasing sequence of right continuous functions $f_{n}, n=1,2, \ldots$, such that $f=\lim _{n \rightarrow \infty} f_{n}$.

From the last remark by an elementary proof we obtain the next assertion.

Remark 3. If a function $f:[a, b) \rightarrow \mathbb{R}$ is right upper semicontinuous, then there is a decreasing sequence of functions $f_{n}:[a, b) \rightarrow \mathbb{R}$ such that

the functions $f_{n}$ are right continuous ;

$f=\lim _{n \rightarrow \infty} f_{n} ;$

all functions $f_{n}, n=1,2, \ldots$, are locally constant from the right, i.e. for each point $x \in[a, b)$ there is a positive real $r_{x, n}$ such that

$$
I_{x, n}=\left[x, x+r_{x, n}\right] \subset[a, b) \text { and } f \uparrow I_{x, n} \text { is constant }
$$

if $\lim \sup _{t \rightarrow x+} f(t)<f(x)$, then for $n$ sufficiently large $f_{n}(x)=f(x)$;

for every integer $n$ the inclusion $\left.f_{n}([a, b)) \subset \operatorname{cl}(f[a, b))\right)$, (where cl denotes the closure operation) holds.

Proof. The set $A$ of all points $x$ at which $\lim \sup _{t \rightarrow x+} f(t)<f(x)$ is countable, i.e. if $A \neq \emptyset$, then $A=\left\{x_{1}, x_{2}, \ldots\right\}$. By Remark 2 there is a decreasing sequence of right continuous functions $g_{n}$ such that $f=\lim _{n \rightarrow \infty} g_{n}$. Fix a positive integer $n$ and observe that there is a sequence of intervals $I_{i, n}=\left[u_{i, n}, v_{i, n}\right)$, $i=1,2, \ldots$, such that:

$$
\begin{aligned}
& {[a, b)=\bigcup_{i} I_{i, n}} \\
& I_{i, n} \cap I_{j, n}=\emptyset \text { for } i \neq j ; \\
& u_{i, n}=x_{i} \text { for } i \leq n ; \\
& \operatorname{osc} g_{n}<\frac{1}{n} \text { on each interval } I_{i, n} ; \\
& g_{n}(x)>f(x) \text { if } x \in I_{i, n} \text { and } i \leq n .
\end{aligned}
$$


Let

$$
h_{n}(x)= \begin{cases}f\left(x_{i}\right) & \text { for } x \in I_{i, n}, \quad i \leq n \\ \sup _{I_{i, n}} g_{n} & \text { for } x \in I_{i, n} \quad i>n .\end{cases}
$$

Then the functions $f_{n}=\min \left(h_{1}, h_{2}, \ldots, h_{n}\right)$, for $n=1,2, \ldots$, satisfy all required conditions.

Theorem 2. If $f=$ a.c. $\lim _{n \rightarrow \infty} f_{n}$, where all functions $f_{n}, n=1,2, \ldots$, are right continuous, then $f$ satisfies the following condition.

(1) For each nonempty perfect set $A \subset[a, b)$ there is an open interval I such that $A \cap I \neq \emptyset$ and the restricted function $f \uparrow(I \cap B)$, where

$$
B=\{x \in A ; x \text { is a right limit point of } A\},
$$

is right continuous at each point of the intersection $B \cap I$.

Proof. Let $A \subset[a, b)$ be a nonempty perfect set and let $B$ denote the set of all right limit points of $A$. For each point $x \in[a, b)$ there is a positive integer $n(x)$ such that $f_{n}(x)=f(x)$ for $n \geq n(x)$. For $n=1,2, \ldots$ put $A_{n}=\{x \in[a, b) ; n(x)=n\}$ and observe that $[a, b)=\bigcup_{n=1}^{\infty} A_{n}$. So there are an open interval $I$ and a positive integer $k$ such that $I \cap B \neq \emptyset$ and $A_{k} \cap I \cap B$ is dense in $B \cap I$. Thus $f(x)=f_{k}(x)$ for each point $x \in I \cap A$ which is a right limit point of $A$ and consequently $f \uparrow(B \cap I)$ is right continuous at each point of $I \cap B$.

The above proof of Theorem 2 is short. However the referee related this statement to the result of Császár and Laczkovich (Theorem 13 of [2], pp. 469) which says that if $X$ is a Baire space, the functions $f_{n}: X \rightarrow \mathbb{R}, n=1,2, \ldots$, are continuous and $f=$ a.c. $\lim _{n \rightarrow \infty} f_{n}$, then the points of discontinuity of $f$ constitute a nowhere dense set in $X$.

The connection between these two results is the following assertion.

Let $(X, \mathcal{T}, \tau)$ be a bitopological space such that $\tau$ is finer than $\mathcal{T}$ and $(X, \mathcal{T})$ is a Baire space. Assume that for every nonempty set $A \in \tau$ there is a nonempty set $B \in \mathcal{T}$ such that $B \subset A$. Then every $\tau$-nowhere dense set is $\mathcal{T}$-nowhere dense and $(X, \mathcal{T})$ is a Baire space.

We arrive at Theorem 2 at once if we observe that the sets $X \subset[a, b)$ having no right isolated points satisfy the conditions of the previous statement. So the quoted theorem of Császár and Laczkovich can be applied. 
Example 1. Let $C$ be the Cantor ternary set and let $I_{n}=\left(a_{n}, b_{n}\right), n=$ $1,2, \ldots$, be an enumeration of all components of the set $[0,1) \backslash C$ such that $I_{n} \cap I_{m}=\emptyset$ for $n \neq m, n, m=1,2, \ldots$ Put

$$
f(x)=\left\{\begin{array}{lll}
1 & \text { for } & x \in B=C \backslash\left\{a_{n} ; n \geq 1\right\} \\
0 & \text { for } \quad x \in[0,1) \backslash B .
\end{array}\right.
$$

Observe that the function $f$ is not of Baire class one. For $n \geq 1$ let

$$
f_{n}(x)=\left\{\begin{array}{lll}
0 & \text { for } & x \in B_{n}=\bigcup_{i \leq n}\left[a_{i}, b_{i}\right) \\
1 & \text { for } \quad x \in[0,1) \backslash B_{n} .
\end{array}\right.
$$

Then all functions $f_{n}, n=1,2, \ldots$, are right continuous, $f_{n} \geq f_{n+1}$ for $n=$ $1,2, \ldots$ and a.c. $\lim _{n \rightarrow \infty} f_{n}=f$.

Now we introduce the following condition $\left(1^{\prime}\right)$.

(1') A function $f$ satisfies condition $\left(1^{\prime}\right)$ if for every nonempty closed set $A \subset[0,1)$ there is an open interval $I$ such that $I \cap A \neq \emptyset$ and the restricted function $f \uparrow(A \cap I)$ is right continuous. (If $x \in A$ is right isolated in $A$, then $f \uparrow A$ is right continuous at $x$ by default.)

Observe that the implication $\left(1^{\prime}\right) \Longrightarrow(1)$ is true. The function $f$ from Example 1 satisfies condition (1) but it does not satisfy condition $\left(1^{\prime}\right)$. Observe also that, by Baire's theorem on Baire 1 functions, every function $f$ satisfying condition $\left(1^{\prime}\right)$ is of Baire 1 class.

Theorem 3. A function $f$ satisfies condition $\left(1^{\prime}\right)$ if and only if it satisfies the following condition.

(2) There is a sequence of nonempty closed sets $A_{n} \subset[a, b)$ such that all restricted functions $f \uparrow A_{n}, n=1,2, \ldots$, are right continuous and $[a, b)=\bigcup_{n=1}^{\infty} A_{n}$.

Proof. $\left(1^{\prime}\right) \Longrightarrow(2)$. We will apply transfinite induction. Let $I_{0}$ be an open interval with rational endpoints such that the restricted function $f \uparrow I_{0}$ is right continuous. Fix an ordinal number $\alpha>0$ and suppose that for every ordinal number $\beta<\alpha$ there is an open interval with rational endpoints $I_{\beta}$ such that $H_{\beta}=I_{\beta} \backslash \bigcup_{\gamma<\beta} I_{\gamma} \neq \emptyset$ and the restricted function $f \uparrow H_{\beta}$ is right continuous. If $G_{\alpha}=[a, b) \backslash \bigcup_{\beta<\alpha} I_{\beta} \neq \emptyset$, then by $\left(1^{\prime}\right)$ there is an open interval $I_{\alpha}$ with rational endpoints such that $I_{\alpha} \cap G_{\alpha} \neq \emptyset$ and the restricted function $f \uparrow\left(I_{\alpha} \cap G_{\alpha}\right)$ is right continuous. Let $\xi$ be the first ordinal number $\alpha$ such that $[a, b) \backslash \bigcup_{\beta<\xi} I_{\beta}=\emptyset$. Since the family of all intervals with rational endpoints is countable, $\xi$ is a countable ordinal number. Every set $H_{\alpha}, \alpha<\xi$, is an $F_{\sigma}$ 
set; so there are closed sets $H_{k, \alpha}, k=1,2, \ldots$, such that $H_{\alpha}=\bigcup_{k=1}^{\infty} H_{k, \alpha}$. Evidently, all restricted functions $f \nmid H_{k, \alpha}, k=1,2, \ldots$ and $\alpha<\xi$, are right continuous. Now enumerate in a sequence $\left(A_{n}\right)$ all sets

$$
H_{k, \alpha}, \quad k=1,2, \ldots \text { and } \alpha<\xi,
$$

and observe that this sequence satisfies all requirements.

$(2) \Longrightarrow\left(1^{\prime}\right)$

Fix a nonempty closed set $A \subset[a, b)$. If $A$ contains isolated points, then condition $\left(1^{\prime}\right)$ is satisfied. So we assume that $A$ is a perfect set. By (2) there is a sequence of closed sets $A_{n}, n=1,2, \ldots$, such that $[a, b)=\bigcup_{n} A_{n}$ and all restricted functions $f\left\lceil A_{n}, n=1,2, \ldots\right.$, are right continuous. Since $A=\bigcup_{n=1}^{\infty}\left(A \cap A_{n}\right)$, there are a positive integer $k$ and an open interval $I$ such that $I \cap A=I \cap A_{k} \neq \emptyset$. But the restricted function $f \uparrow(A \cap I)$ is right continuous; so condition $\left(1^{\prime}\right)$ is satisfied.

Theorem 4. If $f$ satisfies condition ( $1^{\prime}$ ) (or equivalently (2)) from the last theorem, then there is a sequence of functions $f_{n}, n=1,2, \ldots$, which are right continuous and for which a.c. $\lim _{n \rightarrow \infty} f_{n}=f$.

Proof. There is a sequence of nonempty closed sets $A_{n}, n=1,2, \ldots$, such that $[a, b)=\bigcup_{n=1}^{\infty} A_{n}, A_{1} \subset A_{2} \subset \cdots$ and all restricted functions $f \mid A_{n}$, $n=1,2, \ldots$, are right continuous. By Tietze's theorem for $n=1,2, \ldots$ there is a right continuous function $f_{n}:[a, b) \rightarrow \mathbb{R}$ which is equal to $f$ on the set $A_{n}$. Then a.c. $\lim _{n \rightarrow \infty} f_{n}=f$.

Theorem 5. If a function $f$ is upper semicontinuous from the right and satisfies condition ( $\left.1^{\prime}\right)$ (or equivalently (2)), then there is a decreasing sequence of right continuous functions $f_{n}, n=1,2, \ldots$, such that a.c. $\lim _{n \rightarrow \infty} f_{n}=f$.

Proof. Let a function $f$ satisfies the hypothesis of our theorem. There is a sequence of nonempty closed sets $A_{n}, n=1,2, \ldots$, such that $[a, b)=\bigcup_{n=1}^{\infty} A_{n}$ and all restricted functions $f\left\lceil A_{n}, n=1,2, \ldots\right.$, are right continuous. Without loss of the generality we can suppose that $a \in A_{1} \subset A_{2} \subset \ldots \subset A_{n} \subset \ldots$ Fix a positive integer $n$ and enumerate in a sequence $\left(I_{n, k}\right)_{k}$ all components of the set $[a, b) \backslash A_{n}$. If $I_{n, k}=\left(a_{n, k}, b_{n, k}\right), a_{n, k} \in A_{n}$ and $\lim \sup _{t \rightarrow a_{n, k}+} f(t)=f\left(a_{n, k}\right)$, then we find points $c_{n, k, i}, i=1,2, \ldots$, such that

$$
b_{n, k}=c_{n, k, 1}>\ldots>c_{n, k, i}>\ldots \searrow a_{n, k} .
$$

By Theorem 2 and Remark 1 for every $i \geq 1$ there is right constant function $h_{n, k, i}:\left[c_{n, k, i+1}, c_{n, k, i}\right) \rightarrow \mathbb{R}$ such that $h_{n, k, i} \geq f /\left[c_{n, k, i+1}, c_{n, k, i}\right)$ and $h_{n, k, i}\left(c_{n, k, i}\right)<f\left(c_{n, k, i}\right)+\frac{1}{i k}$. Let $g_{n, k, i}(x)=\max \left(h_{n, k, i}(x), f\left(a_{n, k}\right)\right)$ for $x \in$ 
$\left[c_{n, k, i+1}, c_{n, k, i}\right)$. Observe that $g_{n, k, i}\left(\left[c_{n, k, i+1}, c_{n, k, i}\right)\right) \subset \operatorname{cl}\left(f\left(\left[a_{n, k}, b_{n, k}\right)\right)\right), i=$ $1,2, \ldots$ Next in every such interval $I_{n, k}$ we define the function $g_{n, k}$ by

$$
g_{n, k}(x)=g_{n, k, i}(x) \text { for } x \in\left[c_{n, k, i+1}, c_{n, k, i}\right), \quad i=1,2, \ldots
$$

If $I_{n, k}=\left[a_{n, k}, b_{n, k}\right), a_{n, k} \in A_{n}$, and $\limsup _{x \rightarrow a_{n, k}+} f(x)<f\left(a_{n, k}\right)$, then by Remarks 2 and 3 there is a right constant function $h_{n, k}:\left[a_{n, k}, b_{n, k}\right) \rightarrow \mathbb{R}$ such that $h_{n, k} \geq f\left\lceil\left[a_{n, k}, b_{n, k}\right), h_{n, k}\left(a_{n, k}\right)=f\left(a_{n, k}\right)\right.$ and $h_{n, k}\left(\left[a_{n, k}, b_{n, k}\right)\right) \subset$ $\left.\operatorname{cl}\left(f\left(\left[a_{n, k}, b_{n, k}\right)\right)\right)\right)$. Let $g_{n, k}(x)=\max \left(h_{n, k}(x), f(x)\right)$ for $x \in\left[a_{n, k}, b_{n, k}\right)$. Put

$$
g_{n}(x)=\left\{\begin{array}{cl}
f(x) & \text { for } \quad x \in A_{n} \\
g_{n, k}(x) & \text { for } \quad x \in I_{n, k} \quad i, k=1,2, \ldots
\end{array}\right.
$$

Then the function $g_{n}$ is right continuous, $g_{n} \geq f$ and $g_{n} \uparrow A_{n}=f\left\lceil A_{n}\right.$. So, by Lemma 1 we obtain our theorem.

Observe that the last theorem is not a corollary of Theorem 1, since the topology $\tau$ is not $\sigma$-compact.

Acknowledgment I would like to thank to the referee for several suggestions, the reformulation of Theorem 1 and its proof.

Problem. Is Theorem 5 true if we replace condition $\left(1^{\prime}\right)$ by $(1)$ ?

\section{References}

[1] A. M. Bruckner, J. B. Bruckner and B. S. Thomson, Real Analysis, Prentice-Hall International, INC, Upper Saddle River, New Jersey, 1996.

[2] A. Császár and M. Laczkovich, Discrete and equal convergence, Studia Sci. Math. Hungar. 10 (1975), 463-472.

[3] R. J. O'Malley, Approximately differentiable functions. The $r$ topology, Pacific J. Math. 72 (1977), 207-222.

[4] H. L. Royden, Real Analysis, Third Edition, MacMillan, 1988.

[5] B. S. Thomson, Real Functions, Lectures Notes in Math. 1170 (1985), Springer-Verlag. 
